\title{
Novel coding genetic variants of the GBP1 gene in wild and domestic pigs (Sus scrofa)
}

\author{
Shanyuan Chen a , Rui Gomes a , Vânia Costa ${ }^{\text {a }}$, Isabel Rocha a , Attila Zsolnai b,c, István Anton ${ }^{\text {b }}$, \\ Rui Charneca ${ }^{\mathrm{d}}$, Pedro Santos ${ }^{\mathrm{d}}$, José Luis Nunes ${ }^{\mathrm{d}}$, József Buzgó e , Gyula Varga ${ }^{\mathrm{e}}$, \\ Ya-ping Zhang ${ }^{\mathrm{f}}$, Albano Beja-Pereira ${ }^{\mathrm{a}, *}$ \\ a Centro de Investigação em Biodiversidade e Recursos Genéticos da Universidade do Porto (CIBIO-UP), Rua Padre Armando Quintas 7, 4485-661 Vairão, Portugal \\ ${ }^{\mathrm{b}}$ Research Institute for Animal Breeding and Nutrition, H-2053 Herceghalom, Hungary \\ c University of Kaposvár, H-7400, Kaposvár, Hungary \\ ' ICAAM - Instituto de Ciências Agrárias e Ambientais Mediterrânicas, Universidade de Évora, Herdade da Mitra, 7002-554 Évora, Portugal \\ e SEFAG Forest Management and Wood Industry Share Company, Kaposvár, Hungary \\ ${ }^{\mathrm{f}}$ State Key Laboratory of Genetic Resources and Evolution, Kunming Institute of Zoology, Chinese Academy of Sciences, Kunming 650223, China
}

\section{A R T I C L E I N F O}

\section{Article history:}

Received 13 July 2011

Received in revised form 30 January 2012

Accepted 7 February 2012

\section{Keywords:}

Domestic pig

Wild boar

GBP1

Genetic variability

SNP

Rare alleles

\begin{abstract}
A B S T R A C T
The interferon-induced guanylate-binding protein 1 gene (GBP1) plays an important role in host defense against viral, bacterial and protozoan infections. To explore novel genetic variants in this gene, we re-sequenced a 587-bp fragment spanning the exon 2 of the GBP1 gene in a sample panel consisting of 34 wild boars and 59 local domestic pigs from three geographic regions (China, Iberian Peninsula, and Central Europe) and 12 individuals of three commercial breeds (Pietrain, Landrace, and Large White). In a final 543-bp sequence fragment, there were 14 single nucleotide polymorphisms (SNPs), of which five were coding (three novel mutations). A total of 19 haplotypes were reconstructed and most haplotypes were shared by two or more sample groups. Those shared haplotypes revealed a clear signature of genetic introgression from Chinese domestic pigs into European domestic pigs. In addition, there were six haplotypes with frequencies below $1 \%$, but none of them were present in the three commercial breeds (Pietrain, Landrace, and Large White). Although a limited number of individuals and breeds were analyzed, the absence of rare alleles (or haplotypes) in the commercial breeds is an indication that a significant proportion of genetic diversity in domestic species is not present in commercial breeds. This study demonstrated the potential to find sufficient genetic variation for population genetic analyses of demography versus selection, in functional candidate genes of domestic pigs and wild boars worldwide.
\end{abstract}

(c) 2012 Elsevier B.V. All rights reserved.

\section{Introduction}

Interferon (IFN) proteins are essential for host defense against intracellular pathogens and tumor cells, by triggering transcriptional up-regulation of thousands of genes (Borden et al., 2007). Many of the most abundant proteins induced by IFNs are guanosine triphosphatases (GTPases), which constitute four major families - the p47 immunity-related

\footnotetext{
* Corresponding author. Tel.: +351252 660411; fax: +351252661780. E-mail address: albanobp@fc.up.pt (A. Beja-Pereira).
}

GTPases (IRGs), the p65 guanylate-binding proteins (GBPs), Mx proteins, and very large inducible GTPases (VLIG) (MacMicking, 2004; Martens and Howard, 2006).

In recent years, the GBP gene family has received much attention, due to their multiple functions (reviewed in Vestal and Jeyaratnam, 2010). Originally, two members of GBPs (GBP1 and 2) were identified as proteins in human fibroblasts treated with IFNs, particularly IFN- $\gamma$ (Cheng et al., 1983). An early study showed that human GBP1 mediated an antiviral effect against vesicular stomatitis virus and encephalomyocarditis virus (Anderson et al., 1999). Recently, 
extensive transcriptome and genome analyses revealed additional members of GBPs in human (hGBP1 to 7) and mice (mGBP1 to 11) (Kresse et al., 2008; Olszewski et al., 2006). Moreover, recent functional studies also demonstrated that some members of the GBP family (e.g., GBP1, GBP6, GBP7 and GBP10) played important roles in host defense against bacterial and protozoan infections (Degrandi et al., 2007; Kim et al., 2011). Besides those antiviral and antimicrobial activities, members of the GBP family also have other functional effects, such as inhibition of proliferation of endothelial cells, inhibition of matrix metalloproteinase expression, and intestinal epithelial development (Schnoor et al., 2009; Vestal and Jeyaratnam, 2010).

GBP1 is the best studied member of the GBP family and its structure and activity has been well characterized. The structure of human GBP1 contains an N-terminal large G domain, a C-terminal $\alpha$-helical elongated domain, and a short intermediate region connecting domains (Prakash et al., 2000). The large $G$ domain (residues 1 -317) retains the main biochemical properties of the full-length protein and two residues Arg48 and Ser73 located in this domain were found to be crucial for nucleotide-dependent homodimerization and cleavage of GTP or GDP (Ghosh et al., 2006). The availability of structural information, together with the well-characterized functions in immunity, makes the GBP1 gene a good candidate locus for identifying functional genetic variants that potentially underlie disease resistance, in human populations and in economically important livestock species.

Little is so far known about functional genetic variation harbored in the GBP1 gene of major livestock species. Recent analysis of the cDNA sequences of porcine GBP1 gene discovered four single nucleotide polymorphisms (SNPs), including two synonymous mutations each in the exon 8 and the exon 11 and two non-synonymous mutations (in the same codon) resulted in an amino acid change from Lysine to Glycine in the exon 2 (Ma et al., 2008). Additionally, association analysis revealed that those two non-synonymous SNPs in the exon 2 had significant associations with red blood cell traits (Ma et al., 2008). Nevertheless, whether there are quantitative trait loci (QTLs) related to immunity that are co-localized with the GBP1 gene in pig breeds, warrants further study.

Previous genetic studies revealed no indication of an overall reduction in genetic variability of international commercial versus European local pig breeds, at two candidate genes (FABP4 and IGF2) (Ojeda et al., 2006; Ojeda et al., 2008). In contrast, a genome-wide diversity study of worldwide chicken using SNPs demonstrated significant genetic diversity reduction in commercial breeds (Muir et al., 2008). As a result, whether the pattern observed at the FABP4 and IGF2 in domestic pigs can be extrapolated to other candidate genes (e.g., GBP1), remains an open question. In this study, we resequenced a 587-bp fragment spanning the exon 2 of the GBP1 gene in a sample panel consisting of wild boars and local domestic pigs from three geographic regions (China, Iberian Peninsula, and Central Europe) and three commercial breeds, to further explore novel genetic variants harbored in this gene.

\section{Materials and methods}

The sample panel was composed of 105 individuals, including 59 domestic pigs, 34 wild boars, and 12 individuals of three commercial breeds (Table 1). Chinese local pigs were collected from northeastern (five individuals) and northwestern (two individuals) Yunnan, China. Effort was made to collect samples from unrelated individuals. These samples covered three geographic regions - China, Iberian Peninsula, and Central Europe (Hungary and Romania), representing Asian and European lineages or two areas of pig domestication (Giuffra et al., 2000; Larson et al., 2005; Megens et al., 2008).

Genomic DNA was extracted from ear skin tissues using DNeasy Blood \& Tissue Kit (Qiagen $\mathrm{GmbH}$, Hilden, Germany). A 587-bp fragment spanning the exon 2 of the GBP1 gene was amplified by using PCR primer pair Exon 2-SNP-F (5' GGA TAA CAC TTC GGT AAC TTG C 3') and Exon 2-SNP-R (5' GAA GGG GAA ACT GAG ACA CAA T $3^{\prime}$ ), as previously described (Ma et al., 2008). PCR products were purified and sequenced for both strands at the High-Throughput Genomics Unit (HTGU), Department of Genome Sciences, University of Washington (http://www.htseq.org/). The raw sequence trace files were aligned and checked using software package DNASTAR v7.1 (DNASTAR Inc., Madison, WI, USA).

Haplotypes were reconstructed from unphased genotypic sequences by PHASE v2.1.1 algorithm (Stephens and Donnelly, 2003; Stephens et al., 2001), implemented in DnaSP v5.10 (Librado and Rozas, 2009). Diversity measures were calculated in Arlequin v3.5.1.2 (Excoffier and Lischer, 2010). The phylogenetic relations among haplotypes were built by the program Network v4.600 (http://www.fluxusengineering.com/sharenet.htm), using median joining algorithm (Bandelt et al., 1999).

\section{Results and discussion}

After trimming primer sequences, we finally obtained a 543-bp sequence fragment, corresponding to nucleotide positions between 2118 and 2660 of the porcine GBP1 reference sequence NC_010446.4 (15,728 bp, Sscrofa10.2 Primary Assembly). This sequence fragment included $164 \mathrm{bp}$ of the intron 1, $200 \mathrm{bp}$ of the exon 2, and $179 \mathrm{bp}$ of the intron 2 . There were 14 SNPs detected among all sequences

Table 1

Samples of domestic pigs and wild boars analyzed in this study.

\begin{tabular}{llrl}
\hline Sample name & Country & $n$ & Sample group \\
\hline Portuguese Alentejano & Portugal & 26 & dIB \\
Portuguese wild boar & Portugal & 5 & wIB \\
Spanish wild boar & Spain & 12 & wIB \\
Black Slavonian & Hungary & 6 & dHG \\
Swallow-bellied Mangalica & Hungary & 5 & dHG \\
Red Mangalica & Hungary & 5 & dHG \\
Blonde Mangalica & Hungary & 5 & dHG \\
Romanian Mangalica & Romania & 5 & dHG \\
Hungarian wild boar & Hungary & 14 & wHG \\
Chinese local pig & China & 7 & dCN \\
Chinese wild boar & China & 3 & wCN \\
Pietrain & Hungary & 5 & CB \\
Landrace & Hungary & 3 & CB \\
Large White & Hungary & 4 & CB \\
Total & & 105 & \\
\hline
\end{tabular}

Abbreviations: $n$, the number of individuals; dIB, Iberian domestic pigs; wIB, Iberian wild boars; dHG, Hungarian and Romanian domestic pigs; wHG, Hungarian wild boars; dCN, Chinese domestic pigs; wCN, Chinese wild boars; $\mathrm{CB}$, commercial breeds. 
Table 2

The frequency and distribution of 19 haplotypes across sample groups.

\begin{tabular}{|c|c|c|c|c|c|c|c|c|c|c|}
\hline Haplotype & SNPs & $N$ & Frequency & $\mathrm{dIB}$ & wIB & $\mathrm{dHG}$ & wHG & $\mathrm{dCN}$ & wCN & $\mathrm{CB}$ \\
\hline H01 & TCTAAAATCCGGCC & 1 & 0.0048 & 1 & & & & & & \\
\hline H02 & TCTAACCCCCGGCC & 2 & 0.0095 & 2 & & & & & & \\
\hline H03 & TCTAACCTCCGGCC & 4 & 0.0190 & 1 & & & & & 3 & \\
\hline H04 & TCTAAAATGCTACC & 2 & 0.0095 & 1 & & & 1 & & & \\
\hline H05 & СCTGGCCTGCTACC & 4 & 0.0190 & 1 & & 1 & & 2 & & \\
\hline H06 & TCTAACCCGCGGCC & 8 & 0.0381 & 2 & 3 & & 3 & & & \\
\hline H07 & TCTGGCCCGCGGCC & 10 & 0.0476 & 5 & 5 & & & & & \\
\hline H08 & TCTAACCCGCTGCC & 106 & 0.5048 & 20 & 12 & 39 & 12 & 3 & & 20 \\
\hline H09 & TATAACCCGCGGCC & 27 & 0.1286 & 15 & 3 & 9 & & & & \\
\hline $\mathrm{H} 10$ & TCTGGCCCGTTACC & 3 & 0.0143 & 1 & & & 2 & & & \\
\hline H11 & TATAACCCGCGGCT & 1 & 0.0048 & 1 & & & & & & \\
\hline
\end{tabular}

Nucleotide positions of these 14 SNPs corresponding to 2123, 2161, 2216, 2301, 2302, 2314, 2315, 2356, 2502, 2516, 2519, 2526, 2628, and 2641 of the porcine GBP1 gene reference sequence NC_010446.4; Coding SNPs are shaded in gray; $N$, the number of sequences $(N=2 n$, where $n$ is the number of individuals); Abbreviations of sample groups are provided in Table 1.

(Table 2), of which three, five, and six were located in the intron 1, the coding part of the exon 2 (190 bp, corresponding to $1-190 \mathrm{bp}$ of the GBP1 whole coding region sequence), and the intron 2, respectively. Among those five coding SNPs, two non-synonymous mutations 2301A $>G$ and $2302 A>G$ that were equal to $n s 10 A>G$ and $n s 11 A>G$ reported in a previous study (Ma et al., 2008), occurred at the first and second positions of the 4th codon, causing a replacement from Lysine to Glycine (K4G); Two novel mutations $2314 \mathrm{C}>\mathrm{A}$ (non-synonymous) and $2315 \mathrm{C}>\mathrm{A}$ ( synonymous) occurred at the second and third positions of the 8th codon, resulting in a change from Proline to Glutamine (P8Q); The final one novel mutation 2356C $>\mathrm{T}$ (non-synonymous) occurred at the second position of the 22th codon, leading to a substitution from Alanine to Valine (A22V). Notably, high level of genetic variability observed in the GBP1 gene (14 SNPs in 543 bp sequences, $14 / 543=0.026$ ) from this study is comparable to that found in the FABP4 gene (134 SNPs in 6400 bp sequences, $134 / 6400=0.021$ ) (Ojeda et al., 2006).
A total of 19 haplotypes were reconstructed by PHASE analysis from these 14 SNPs (Table 2). The predominant haplotype H08 (identical to the reference sequence NC_010446. 4) with a frequency of $50.48 \%$ was distributed in all sample groups except for Chinese wild boars ( $\mathrm{wCN}$ ); while the second common haplotype H09 had a frequency of $12.86 \%$, found in three sample groups (dIB, wIB, and dHG) (Table 2 and Fig. 1). Most of the haplotypes were shared by two or more sample groups, except for seven unique haplotypes (Table 2 and Fig. 1). Those shared haplotypes revealed a clear signature of genetic introgression from Chinese domestic pigs (dCN) into European domestic pigs ( $\mathrm{dIB}$ and dHG). For instance, the haplotypes $\mathrm{H} 05$ and $\mathrm{H} 08$ were shared by dCN, dIB, and dHG, while the haplotypes H16 and H17 were shared by dCN and dHG. The genetic contribution of Chinese domestic pigs to European domestic pigs and perhaps indirectly to commercial breeds detected here, was congruent with historical records and previous genetic studies (e.g., Giuffra et al., 2000).

As expected, Chinese domestic pigs ( $\mathrm{dCN}$ ) displayed highest values of both haplotype diversity and nucleotide diversity, whereas the three commercial breeds pooled together (CB) showed lowest value of haplotype diversity (Table 3). Within Europe, both domestic pigs (dIB) and wild boars (wIB) from the Iberian Peninsula exhibited higher levels of genetic variability than those (dHG and wHG) from Central Europe (Hungary and Romania). This pattern of diversity distribution observed here was congruent with previous studies that revealed larger overall genetic variability in Asian (or Chinese) domestic pigs and wild boars than their European counterparts (Megens et al., 2008; Ojeda et al., 2008).

In summary, we detected three novel coding SNPs (two non-synonymous and one synonymous) in the coding part of the GBP1 exon 2 (residues 1-63), which belongs to the large $G$ domain (residues 1-317) of the GBP1 protein (Ghosh et al., 2006; Prakash et al., 2000). Although our sequenced coding fragment contains seven GTP/ $\mathrm{Mg}^{2+}$ binding sites (residues 47-53), both newly discovered nonsynonymous mutations ( $\mathrm{P} 8 \mathrm{Q}$ and $\mathrm{A} 22 \mathrm{~V}$ ) are located at upper positions of this binding domain. Thus, whether the two mutations would have structural and functional effects, needs to be further explored. In addition, we found six

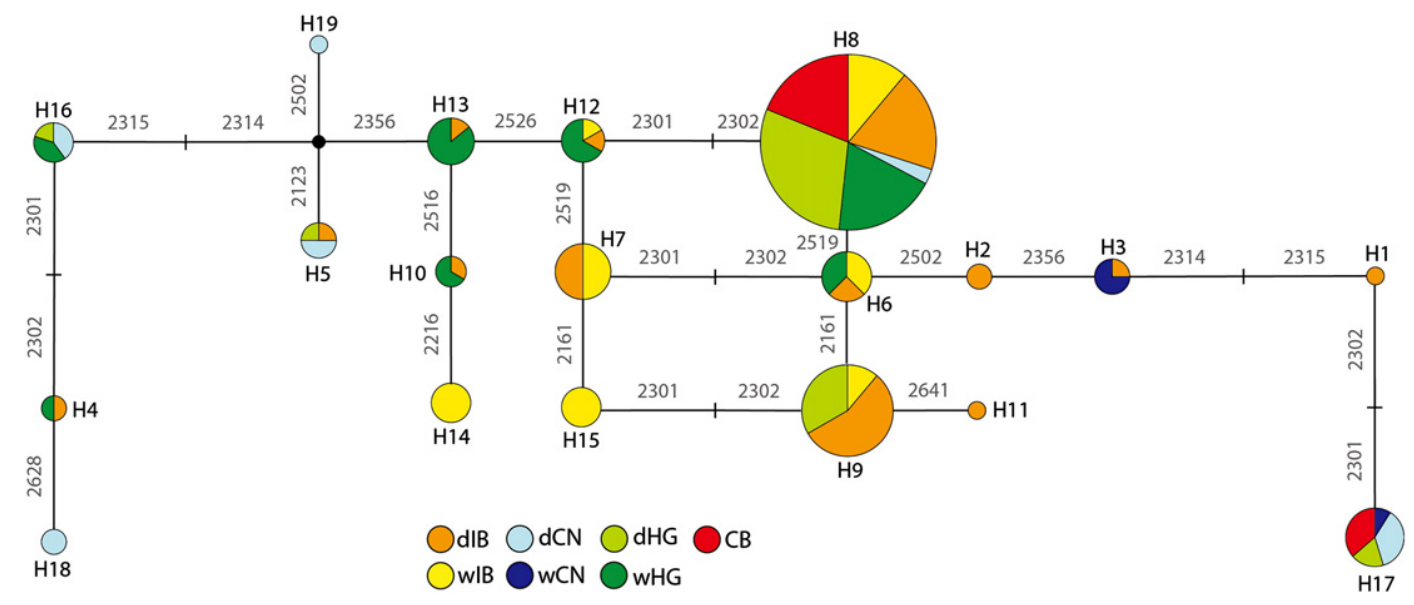

Fig. 1. The median-joining network of the 543-bp GBP1 haplotypes. The size of each circle is proportional to its frequency in the number of sequences. Abbreviations for sample groups are provided in Table 1. 
Table 3

Diversity measures across sample groups.

\begin{tabular}{lrrll}
\hline Sample group & \multicolumn{1}{l}{$N$} & \multicolumn{1}{l}{$S$} & $h$ & $\pi$ \\
\hline dIB & 52 & 12 & 0.7685 & 0.0041 \\
wIB & 34 & 7 & 0.8182 & 0.0049 \\
dHG & 52 & 10 & 0.4133 & 0.0027 \\
wHG & 28 & 8 & 0.7593 & 0.0037 \\
dCN & 14 & 10 & 0.8681 & 0.0081 \\
wCN & 6 & 7 & 0.7333 & 0.0074 \\
CB & 24 & 7 & 0.2899 & 0.0037 \\
All & 210 & 14 & 0.7208 & 0.0050 \\
\hline
\end{tabular}

$N$, the number of sequences; $S$, number of polymorphic sites; $h$, haplotype diversity; $\pi$, nucleotide diversity per site. Abbreviations of sample groups are provided in Table 1.

haplotypes with frequencies below $1 \%$, but none of them were present in the three commercial breeds (Pietrain, Landrace, and Large White). Although a limited number of individuals and breeds were analyzed, the absence of rare alleles (or haplotypes) in the commercial breeds is an indication that a significant proportion of genetic diversity in domestic species is not present in commercial breeds (Muir et al., 2008). Furthermore, this study demonstrated the potential to find sufficient genetic variation for population genetic analyses of demography versus selection, in functional candidate genes of domestic pigs and wild boars worldwide. This study also highlighted the urgent needs of applying resequencing techniques such as next-generation sequencing technology to investigate genome-wide functional genetic variation in wild boars and local domestic pigs worldwide.

\section{Conflict of interest statement}

The authors have no conflict of interests regarding the research reported in this manuscript.

\section{Acknowledgments}

We thank two anonymous reviewers for their constructive comments. This work was funded by Fundação para a Ciência e Tecnologia (FCT) project PTDC/CVT/68907/2006 (AB-P). SC is supported by a FCT individual fellowship grant SFRH/BPD/46082/2008. We thank Vlado Margeta for his help in sampling Hungary Black Slavonian breed.

\section{References}

Anderson, S.L., Carton, J.M., Lou, J., Xing, L., Rubin, B.Y., 1999. Interferon-induced guanylate binding protein-1 (GBP-1) mediates an antiviral effect against vesicular stomatitis virus and encephalomyocarditis virus. Virology 256, 8-14.

Bandelt, H.J., Forster, P., Rohl, A., 1999. Median-joining networks for inferring intraspecific phylogenies. Mol. Biol. Evol. 16, 37-48.

Borden, E.C., Sen, G.C., Uze, G., Silverman, R.H., Ransohoff, R.M., Foster, G.R. Stark, G.R., 2007. Interferons at age 50: past, current and future impact on biomedicine. Nat. Rev. Drug Discov. 6, 975-990.

Cheng, Y.S., Colonno, R.J., Yin, F.H., 1983. Interferon induction of fibroblast proteins with guanylate binding activity. J. Biol. Chem. 258, 7746-7750.
Degrandi, D., Konermann, C., Beuter-Gunia, C., Kresse, A., Würthner, J., Kurig, S., Beer, S., Pfeffer, K., 2007. Extensive characterization of IFN-induced GTPases mGBP1 to mGBP10 involved in host defense. J. Immunol. 179, 7729-7740.

Excoffier, L., Lischer, H.E.L., 2010. Arlequin suite ver 3.5: a new series of programs to perform population genetics analyses under Linux and Windows. Mol. Ecol. Resour. 10, 564-567.

Ghosh, A., Praefcke, G.J.K., Renault, L., Wittinghofer, A., Herrmann, C., 2006. How guanylate-binding proteins achieve assembly-stimulated processive cleavage of GTP to GMP. Nature 440, 101-104.

Giuffra, E., Kijas, J.M., Amarger, V., Carlborg, O., Jeon, J.T., Andersson, L., 2000. The origin of the domestic pig: independent domestication and subsequent introgression. Genetics 154, 1785-1791.

Kim, B.-H., Shenoy, A.R., Kumar, P., Das, R., Tiwari, S., MacMicking, J.D., 2011. A family of IFN- $\gamma$-inducible $65-\mathrm{kD}$ GTPases protects against bacterial infection. Science 332, 717-721.

Kresse, A., Konermann, C., Degrandi, D., Beuter-Gunia, C., Wuerthner, J., Pfeffer, K., Beer, S., 2008. Analyses of murine GBP homology clusters based on in silico, in vitro and in vivo studies. BMC Genomics 9, 158.

Larson, G., Dobney, K., Albarella, U., Fang, M., Matisoo-Smith, E., Robins, J., Lowden, S., Finlayson, H., Brand, T., Willerslev, E., Rowley-Conwy, P., Andersson, L., Cooper, A., 2005. Worldwide phylogeography of wild boar reveals multiple centers of pig domestication. Science 307, 1618-1621.

Librado, P., Rozas, J., 2009. DnaSP v5: a software for comprehensive analysis of DNA polymorphism data. Bioinformatics 25, 1451-1452.

Ma, G., Huang, J., Sun, N., Liu, X., Zhu, M., Wu, Z., Zhao, S., 2008. Molecular characterization of the porcine GBP1 and GBP2 genes. Mol. Immunol. 45, 2797-2807

MacMicking, J.D., 2004. IFN-inducible GTPases and immunity to intracellular pathogens. Trends Immunol. 25, 601-609.

Martens, S., Howard, J., 2006. The interferon-inducible GTPases. Annu. Rev. Cell Dev. Biol. 22, 559-589.

Megens, H.-J., Crooijmans, R.P.M.A., San Cristobal, M., Hui, X., Li, N., Groenen, M.A.M., 2008. Biodiversity of pig breeds from China and Europe estimated from pooled DNA samples: differences in microsatellite variation between two areas of domestication. Genet. Sel. Evol. 40, 103-128.

Muir, W.M., Wong, G.K.-S., Zhang, Y., Wang, J., Groenen, M.A.M., Crooijmans, R.P.M.A., Megens, H.-J., Zhang, H., Okimoto, R., Vereijken, A., Jungerius, A., Albers, G.A.A., Lawley, C.T., Delany, M.E., MacEachern, S., Cheng, H.H., 2008. Genome-wide assessment of worldwide chicken SNP genetic diversity indicates significant absence of rare alleles in commercial breeds. Proc. Natl. Acad. Sci. 105, 17312-17317.

Ojeda, A., Rozas, J., Folch, J.M., Pérez-Enciso, M., 2006. Unexpected high polymorphism at the FABP4 gene unveils a complex history for pig populations. Genetics 174, 2119-2127.

Ojeda, A., Huang, L.-S., Ren, J., Angiolillo, A., Cho, I.-C., Soto, H., Lemús-Flores, C., Makuza, S.M., Folch, J.M., Pérez-Enciso, M., 2008. Selection in the making: a worldwide survey of haplotypic diversity around a causative mutation in porcine IGF2. Genetics 178, 1639-1652.

Olszewski, M.A., Gray, J., Vestal, D.J., 2006. In silico genomic analysis of the human and murine Guanylate-Binding Protein (GBP) gene clusters. J. Interferon Cytokine Res. 26, 328-352.

Prakash, B., Praefcke, G.J.K., Renault, L., Wittinghofer, A., Herrmann, C., 2000. Structure of human guanylate-binding protein 1 representing a unique class of GTP-binding proteins. Nature 403, 567-571.

Schnoor, M., Betanzos, A., Weber, D.A., Parkos, C.A., 2009. Guanylate-binding protein-1 is expressed at tight junctions of intestinal epithelial cells in response to interferon-gamma and regulates barrier function through effects on apoptosis. Mucosal Immunol. 2, 33-42.

Stephens, M., Donnelly, P., 2003. A comparison of Bayesian methods for haplotype reconstruction from population genotype data. Am. J. Hum. Genet. 73, 1162-1169.

Stephens, M., Smith, N.J., Donnelly, P., 2001. A new statistical method for haplotype reconstruction from population data. Am. J. Hum. Genet. 68, 978-989.

Vestal, D.J., Jeyaratnam, J.A., 2010. The guanylate-binding proteins: emerging insights into the biochemical properties and functions of this family of large interferon-induced guanosine triphosphatase. J. Interferon Cytokine Res. 31, 89-97. 